\title{
COMPUTER-GUIDED FRONTAL BONE DEFECT RECONSTRUCTION USING CUSTOMIZED THREE DIMENSIONAL PEEK-PSI
}

\author{
Mostafa Shindy*, Khaled Amr * and Hussein Hatem **
}

\begin{abstract}
Purpose : Cranio-facial trauma with Frontal bone defects develops a frustrating facial deformity with a resultant obvious bad appearance. Conventional frontal defect reconstruction often develops an asymmetric facial contour due to the lack of ultimate accuracy. This study aimed to utilize the computer guided 3D planning, designing and printing technology to reconstruct frontal bone defects using Poly-ether-ether-ketone (PEEK) material to reach a more precise prosthesis for better frontal bone contour and esthetics.
\end{abstract}

Patients and methods: A total of six patients suffering from Frontal bone defects were included in this study from the department of oral and maxillofacial surgery, faculty of Dentistry, Cairo University. All the patients were subjected to clinical and CT radiographic examination to assess the extent of the defect and its implications. The computer software was used to manipulate the CT data to create a skull template and an anatomically precise prosthesis through "mirroring" using the sound contra-lateral skull as a reference. The prosthetic part was 3D printed with PEEK-patient specific implant (PSI) material and was adapted and fixed in place through either coronal incision, in four patients, or the pre-existing frontal scars, in two patients. After a 12 months follow-up, clinical inspection and palpation was performed. Postoperative CT imaging was done to validate the accuracy of the implanted PEEK-PSI part in relation to the preoperative computerized plan.

Results: A male predominance in all cases with a mean age of 46 years was found with a dominating etiology of motor vehicle accidents (MVA). The surgeries in all cases proceeded uneventful. Surgical site healing was normal without any signs of infection. The anatomic frontal contour and facial esthetics were admirable. The CT scan revealed the precise adaptation of the prosthesis in place without any pathology. Preoperative virtual plan fell precisely in place when compared with the post-surgical CT images after the prosthesis fixation. Conclusion: Computer guided 3D designing and printing of the prosthesis in frontal defect reconstruction was highly beneficial; higher accuracy and less obtained surgical errors due to the perfect fit of the prosthesis, along with the resultant reduction of the surgical time was documented. The integration of computer programs in surgery planning and custom prosthesis designing in defect reconstruction is highly advisable due to its great benefits and accuracy.

Keywords: Frontal Defect, Computer-guided reconstruction, PEEK.

\footnotetext{
* Lecturer of Oral and Maxillofacial Surgery, Oral and Maxillofacial Surgery Department, Faculty of Dentistry, Cairo University.

** Associate Professor of Oral and Maxillofacial Surgery, Oral and Maxillofacial Surgery Department, Faculty of Dentistry, Cairo University.
} 


\section{INTRODUCTION}

The majority of frontal fractures are the result of high-velocity injuries, hence, their often concomitant association with head and neck or spine injuries. Patients suffering from injuries to the frontal bone are often victims of polytrauma due to its advantageous anatomy which dissipates minor blow forces without causing its fracture which explains the low incidence of frontal bone injuries in relation to all other facial bones $(2-15 \%)^{(1)}$.

Frontal defects develop marked obvious esthetic deformities which need meticulous and accurate management. The main treatment goal remains to be the esthetic re-establishment of a symmetrical facial contour ${ }^{(2-3)}$.

Major craniofacial defects, especially frontal defects, resulting from trauma or tumors, present a challenge to reach an ideal esthetic facial symmetry and reconstruction accuracy ${ }^{(4)}$.

The complex individual frontal contour and orbital roof connection along with the obvious contra-lateral side contour to compare, offered a great difficulty to attain the exact duplication by the surgeons. The conventional and manual symmetric precise reconstruction of the craniofacial defects remains a tricky issue for all the surgeons ${ }^{(5)}$.

Computerized tomography (CT) has been long used as medical tool to diagnose different craniofacial defects. The introduction of the advanced computer software algorithms allowed the interesting CT image reformatting to accurately define and virtually reconstruct such defects ${ }^{(6)}$.

This is done nowadays through the computerized preoperative mirroring of the healthy side to use it as a template to fabricate an ideal prosthesis, using various materials, for facial symmetry production $^{(7)}$.

Computer designed "mirrored" templates provided a huge advancement and improvement in the process of esthetic facial reconstruction to become an irreplaceable surgical armamentarium ${ }^{(4,8)}$.
Various materials have long been used for closure and reconstruction of cranio-facial defects including autogenous bone grafts, silicone, titanium mesh, porous hydroxyl-apatite and poly-ether-etherketone (PEEK) ${ }^{(9-10)}$.

PEEK is a highly inert smooth-surfaced semicrystalline poly aromatic polymer which offers a great deal of strength and environmental durability. This biomaterial was originally used in orthopedics in cervical disc problems ${ }^{(10-12)}$.

In this study, PEEK-PSI was used for frontal reconstruction due to its high resistance to biological degradation with the advantage of being easily customizable to any defect geometry ${ }^{(13-14)}$.

This study presents the clinical application of CT/3D designing of the skull template to design and print the prosthesis geometry, using PEEK-PSI material, needed for the frontal defect reconstruction in an attempt to minimize human errors and enhance the esthetic accuracy to reach a flawless facial profile.

\section{PATIENTS AND METHODS}

This study included a total of six male patients who were selected from the department of oral and maxillofacial surgery, faculty of Dentistry, Cairo University.

They all had an obvious post-traumatic frontal bone defect which highly affected the facial esthetics of the patient and required surgical reconstruction to reach an ideal esthetic facial profile. A written consent and an ethical clearance were obtained from each patient. PEEK implant material was used in all cases to restore the anatomic contour of the area.

\section{Exclusion criteria:}

- An obvious soft tissue defect which would hinder the post-reconstruction site closure.

- Infection and/or systemic diseases as Diabetes Mellitus. 


\section{Preoperative assessment:}

History of the trauma was documented. The patients had a mean age of 46 years.

Clinical examination was performed to evaluate the defect and the pre-exited facial scars with the attention to the original facial contour of the contralateral side. (Fig. 1)

Preoperative CT images were done to identify the exact defect extensions. (Fig. 2)

\section{Processing Method of PEEK-PSI prosthesis:}

Preoperative digitalized three-dimensional CT was obtained from axial and coronal images. The software was used to manipulate the CT data to create a skull template through "mirroring" using the sound contra-lateral skull use as a reference from the CT database to be scaled to fit and cover the defect for reconstruction of the defect. When the reference template is well fitted to the target skull, the shape of the reference template is used to build an anatomically precise prosthesis for the final craniofacial reconstruction.

Skull and implant models were studied and approved. Images were sent to be printed by the manufacturer who sent the final definitive part to be used. (Fig. 3)

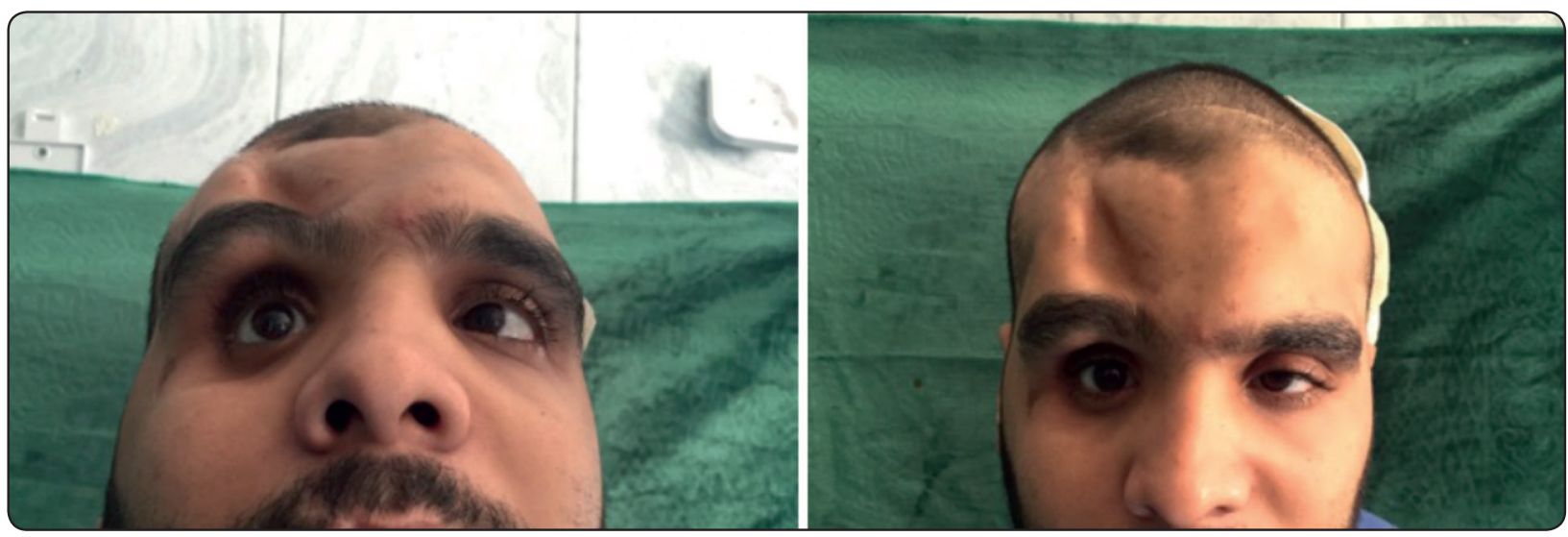

Fig. (1) Photographs showing the preoperative frontal defect.

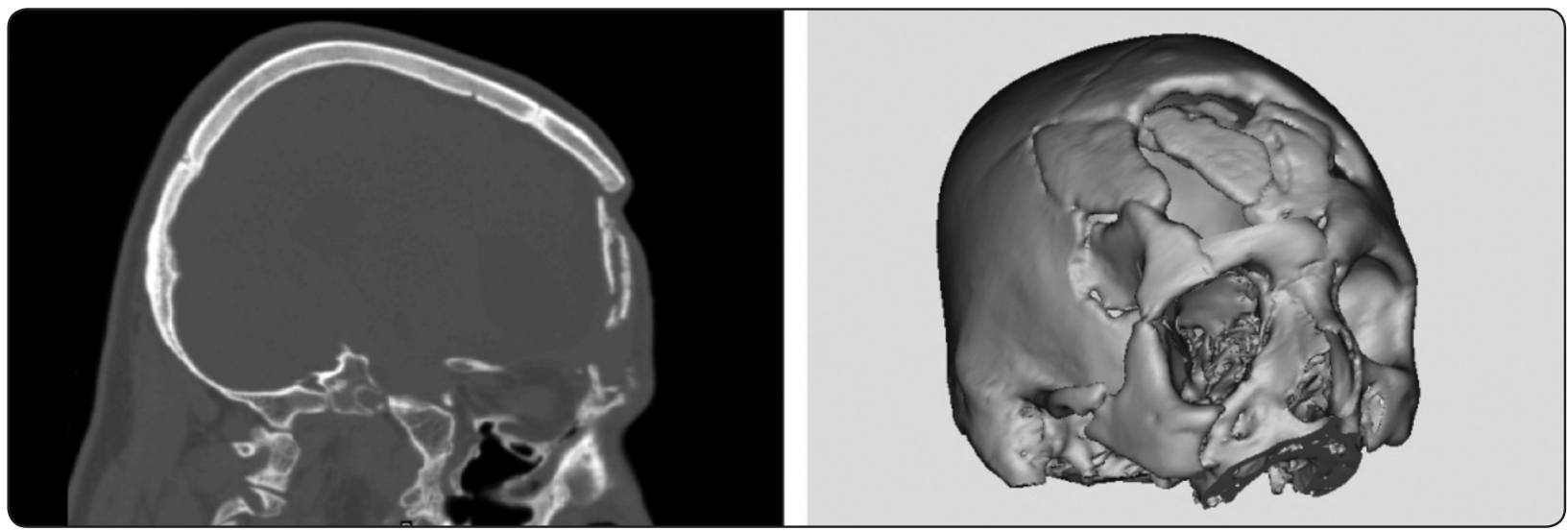

Fig. (2) CT images showing mid-face fractures associated with orbital fracture. 


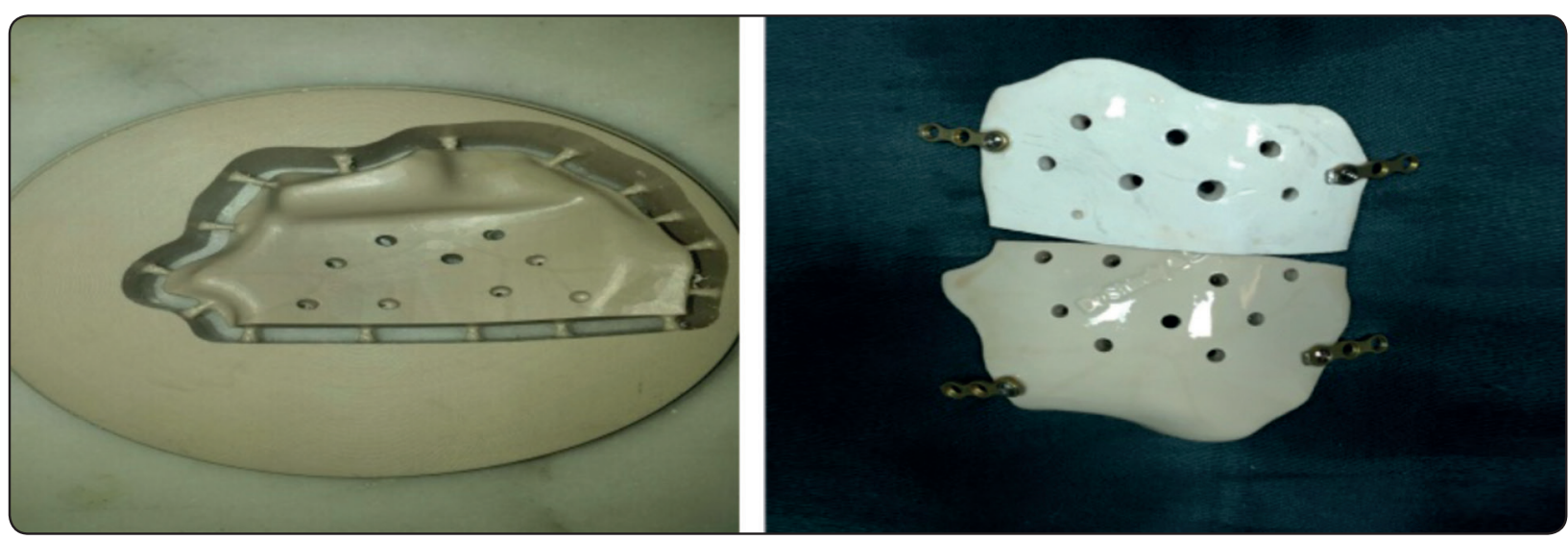

Fig. (3) Photographs showing the printed PEEK prosthetic implant.

The patients underwent the surgeries under general anesthesia. Four patients needed coronal incision to adequately explore and manage the defect, while, the other two were approached through their old pre-existing frontal scars. (Fig. 4)

The custom fabricated PEEK prosthetic part was adapted in the computerized pre-determined place and tested to cover the bone-defect margins. It was then fixed in position with 2.0 titanium mini-plates and screws according to the requirements of each case. (Fig. 4)
Tissue repositioning and suturing were performed. The esthetic contour was immediately regained. (Fig. 5)

Immediate postoperative close monitoring was mandatory. Follow up of the patients continued for 12 months.

Postoperative clinical examination through inspection and palpation was performed.CT imaging was done to validate the implanted PEEK prosthetic part. The comparison between the preoperative computer planning and the postoperative outcome was performed.

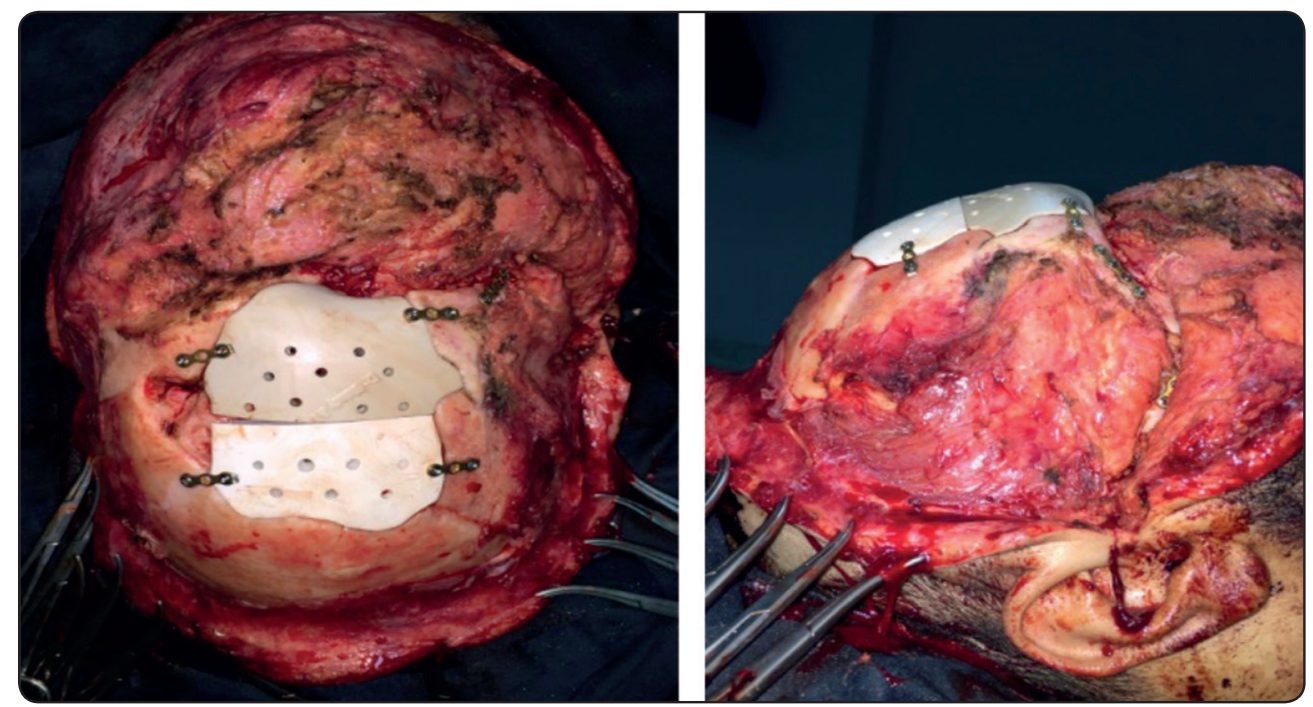

Fig. (4) Photographs showing the intra-surgical adaptation and fixation of the prosthesis. 


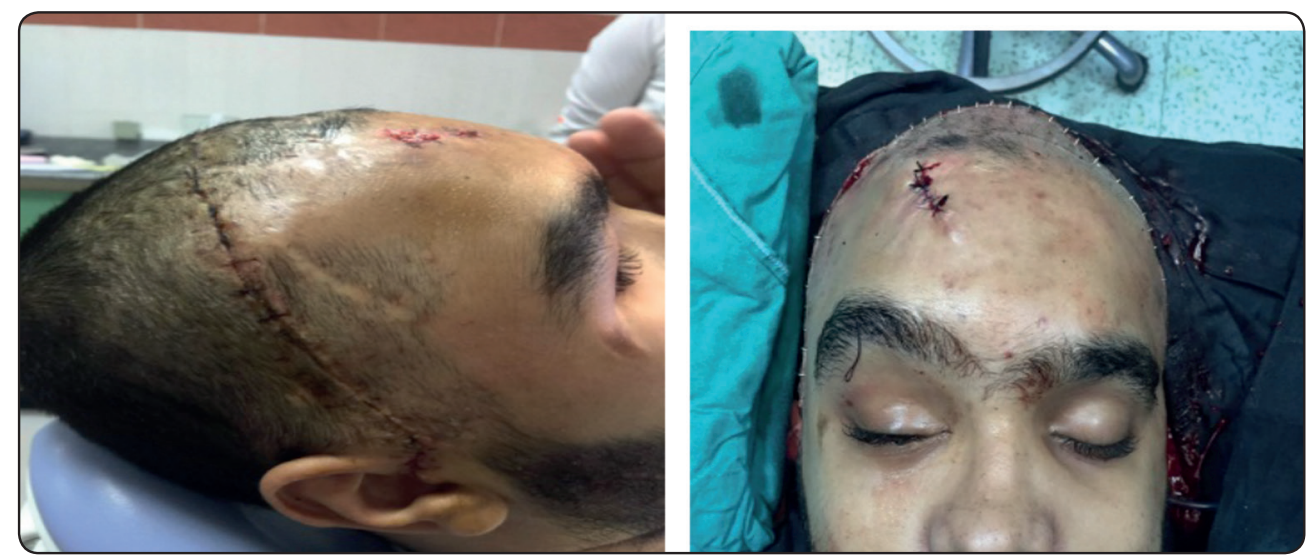

Fig. (5) A photograph showing the immediate post-surgical contour regain.

\section{RESULTS}

A male predominance in all cases was with a mean age of 46 years with a dominating etiology of motor vehicle accidents (MVA) was found.

Various forms of concomitant injuries and maxillofacial fractures were documented irrelevant to the defect under study.

All surgeries were successful and the resultant postoperative pain and swelling were tolerable and considered normal.

The healing in all cases proceeded uneventful. All patients were clinically content and satisfied.
A twelve months follow-up period after the surgical intervention confirmed the maintenance of the anatomic frontal contour and facial esthetics of the area.

Surgical site healing was normal and there were no signals of infection in the PEEK-PSI part.

Also, CT scan revealed the adaptation of the prosthesis in place with the bone without any resorption or pathological repercussions. Preoperative virtual plan fell precisely in place when compared with the post-surgical CT images after the prosthesis fixation. (Fig. 6)

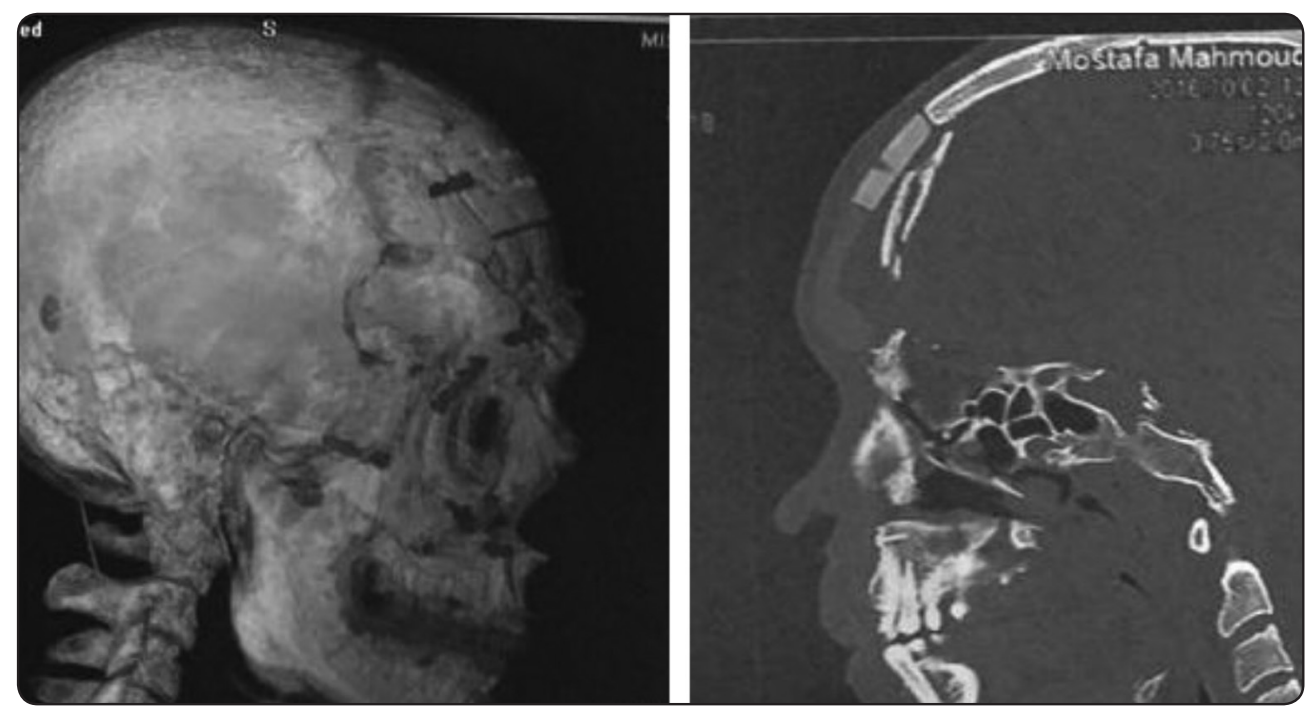

Fig. 6: CT images revealing the precise fit of the prosthesis in accurate position. 


\section{DISCUSSION}

Many management schemes of frontal bone defects are presented in the literature to remain a controversial issue. However, surgeons agreed upon the main objectives of such managements; the prevention of infection of the intra-cranial components and the re-establishment of the frontal contour and esthetics ${ }^{(15-17)}$.

Various types of autogenous and alloplastic materials were used in the literature for maxillofacial reconstruction. The golden standards were attributed to autogenous bone grafts, however, with the disadvantages of local resorption and donor site morbidity issues ${ }^{(10)}$.

Alloplastic implants with computerized designs allowed for a break-through in the concept of maxillofacial reconstruction and have become a dependable alternative ${ }^{(4)}$.

PEEK is a well established material in nonmedical fields. Despite the few reports on its use in the reconstruction of maxillofacial defects, recent studies documented its successful use ${ }^{(16)}$.

In this study, PEEK-PSI was used for the reconstruction of the presented frontal defects. Throughout the follow up period, all cases showed the normal surgical site healing without any signs of infection in the PEEK prosthetic part, which held its shape without resorption or alteration.

This came in accordance with Scolozzi et al ${ }^{(4)}$ and Hanasono et $\boldsymbol{a l}^{\left({ }^{(10)}\right.}$ who attributed its successful use to many factors including its great handling properties, biocompatible durability, strength and similar stiffness to cortical bone.

In addition, Kim et $_{\text {al }}{ }^{(10)}$ documented the excellent postoperative esthetic and functional results in 2009 in their case series of complex maxillofacial defects reconstruction.

In a 6 year review, AbdullaKutty et al ${ }^{(18)}$ declared the biological degradation resistance of PEEK along with its high strength and stiffness.
Nowadays, the introduction of computer software in the surgical steps planning and in the process of fabrication of customized prosthesis in defect reconstruction have been focused on in an attempt to increase the accuracy and eliminate human errors along with the attempt to anticipate any unexpected intra-surgical problems ${ }^{(6,19-20)}$.

This study presented the clinical cases of frontal defect reconstruction using the computer guided 3D/ CT imaging through an employed reference skull template to design and produce a precise prosthesis. The computer tools were used in the evaluation of the defects, custom prosthesis design and printing, surgery planning and reconstruction of the defect.

In this study, the customized prosthesis fitted accurately in place in all cases with the evident intra-operative ease of adaptation and fixation. Postoperative clinical esthetic facial profiles of the patients were admirable and considered normal.

Moreover, the comparison between the virtual computerized preoperative plan and the postoperative performed $\mathrm{CT}$ images after the prosthesis fixation were precise. This was attributed to the successful and accurate computerized format of the created skull template and the prosthesis design. The 3D printing and the skull model of the patient have allowed a clear visualization of the defect area; the operation time was reduced as well as a satisfactory result was obtained by using this designing method.

This was in agreement with Singare et al ${ }^{(6)}$ who acknowledged the benefits of 3D designing and printing in the maxillofacial field. He successfully employed the computer software in the reconstruction of frontal defects using the biomaterial calcium phosphate cement (CPC) customized prosthesis. Furthermore, Camarini et al ${ }^{(16)}$ successfully used computerized custom prosthesis in frontal bone defect reconstruction.

For these reasons, recent studies highly support the dependability on the computer software in surgery planning and executing, with their urge in more computerized surgery implementation. 


\section{CONCLUSION AND SIGNIFICANCE}

The results of this study affirmed the benefits of the computer guided 3D designing and printing of the prosthesis used for the frontal defect reconstruction. These included the higher accuracy, the less obtained errors during surgery, and the perfect fit of the prosthesis, along with the resultant reduction of the surgical time.

The integration of computer programs in surgery planning and custom prosthesis designing and printing in maxillofacial defect reconstruction is highly advisable due to its great benefits and accuracy.

The need to include different materials for reconstruction in a future study is evident in order to verify differences on a biomechanical level.

\section{REFERENCES}

1. Gossman DG, Archer SM and Arosarena O. Management of frontal sinus fractures: a review of 96 cases. Laryngoscope; 116: 1357, 2006.

2. Stanley RB: Fractures of the frontal sinus. Clin Plast Surg; 16:115, 1989.

3. Wallis A and Donald PJ: Frontal sinus fractures: a review of 72 cases. Laryngoscope; 98:593, 1988.

4. Scolozzi P. Maxillofacial reconstruction using PolyetheretherKetone Patient-Specific implants by "Mirroring" Computational Planning. Aesthetic Plastic Surgery; 36: 660, 2012.

5. Luo J, Morrison AD, Hayes AJ, Bala A and Watts G. single piece Titanium plate Cranioplasty reconstruction of complex defects. J Craniofacial Surgery; 29: 839, 2018.

6. Singare $\mathrm{S}$, Shenggui $\mathrm{C}$ and Nan Li. The benefit of 3D printing in medical field: Example frontal defect reconstruction. J material Sci Eng; 6: 2, 2017.

7. Markiewicz MR and Bell RB. The use of 3D imaging tools in facial plastic surgery. Facial plastic surgery clinics of North America; 19: 655, 2011.

8. Morrison DA, Guy DT, Day RE, Lee GY. Simultaneous repair of two large cranial defects using rapid prototyping and custom computer-designed titanium plates: a case report. Proc Inst Mech Eng H; 225: 1108, 2011.

9. Neovius E and Engstrand T. Craniofacial reconstruction with boe and biomaterials: Review over the last 11 years. J Plastic Reconstructive Aesthetic Surgery; 64: 834, 2011.

10. Kim MM, Boahene KD and Byrne PJ. Use of customized Poly-ether-ether-Ketone (PPEK) implants in the reconstruction of complex maxillofacial defects. Arch Facial Plast Surg; 11: 53, 2009.

11. Hanasono MM, Goel N, Demonte F. Calvarial reconstruction with polyetheretherketone implants. Ann Plast Surg; 62:653, 2009.

12. Nieminen T, Kallela I, Wuolijoki E, et al. Amorphous and crystalline polyetheretherketone: mechanical properties and tissue reactions during a 3-year follow-up. J Biomed Mater Res A; 10:377, 2007.

13. Kurtz SM, Devine JN. Peek biomaterials in trauma, orthopedic, and spinal implants. Biomaterials; 28: 4845, 2007.

14. Ponnappan RK, Serhan H and Zarda B et al. Biomechanical evaluation and comparison of polyetheretherketone rod system to traditional titanium rod fixation. Spine J; 9:263, 2009.

15. Gabrielli Mfr, Gabrielli Mac, and Vieira Eh, et al. IMMEDIATE reconstruction of frontal sinus fractures: review of 26 cases. J Oral Maxillofac Surg; 62:582, 2004.

16. Camarini ET, Tomech JK, Dias RR and Jose da Silva E. Reconstruction of frontal bone using specific implant Polyether-Ether-Ketone. J Craniofac Surg; 22: 2205, 2011.

17. Bell BR. Management of frontal sinus fractures. Oral Maxillofacial Surg Clin; 21: 227, 2009.

18. AbdullaKutty A, Madhavarajan S, Collyer J and Sneddon K. Uses of Peek in maxillofacial reconstruction: a 6 year review of cases. British J Oral Maxillofac Surgery; 4: 249, 2012.

19. Eppley BL, Kilgo M, Coleman JJ. Cranial reconstruction with computer-generated hard-tissue replacement patientmatched implants: indications, surgical technique, and long-term follow-up. Plast Reconstr Surg; 109:864, 2002.

20. Mustafa SF, Evans PL, Bocca A, Patton DW and Sugar AW et al. Customized titanium reconstruction of post-traumatic orbital wall defects: a review of 22 cases. International journal of oral and maxillofacial surgery; 40: 1357, 2011. 